Proceedings of SALT 26: 913-933, 2016

\title{
Subjective attitudes and counterstance contingency *
}

\author{
Christopher Kennedy \\ University of Chicago
}

\author{
Malte Willer \\ University of Chicago
}

\begin{abstract}
Across languages, Subjective Attitude Verbs (SAVs), such as English find, differ from ordinary doxastic attitude verbs (such as English believe) in that they require their complement to be subjective in a particular way. The goal of this paper is to develop a semantics for SAVs that predicts this fact but also captures the finer-grained differences between find-type SAVs and consider-type SAVs that make the former more restrictive than the latter. We propose that in terms of their core, at issue content, SAVs are just like believe in expressing a doxastic attitude towards the prejacent. They differ in that they introduce a presupposition that their prejacent is contingent with respect to a distinct set of discourse alternatives that we label COUNTERSTANCES: alternative common grounds that differ only in decisions about how to resolve semantic underdetermination. The larger theoretical significance of our proposal is that it supports a characterization of 'subjective language' as an essentially pragmatic, context-sensitive phenomenon, which does not correlate with semantic type (pace Sæbø) but derives from speakers' recognition of the possibility of counterstance.
\end{abstract}

Keywords: subjective attitude verbs, subjective predicates, personal taste, attitude ascriptions, discourse alternatives, presupposition, semantic indeterminacy

\section{Introduction}

Sæbø (2009) examines SUBJECTIVE ATTITUdE VERBs, such as English find, with the goal of adjudicating between different formal accounts of the semantics of taste predicates, evaluative adjectives, and other expressions with 'subjective' content (see also Borkin 1974; Mitchell 1986; Stephenson 2007; Bouchard 2012; Fleisher 2013; Kennedy 2013; Hirvonen 2014; Bylinina forthcoming). Find is notable because it requires its complement to be subjective in a particular way. Thus (1a) with the evaluative adjective fascinating is acceptable, but (1b) with vegetarian is not, even though this is an expression for which there may be inter-speaker variation as to which criteria are relevant for determining whether the predicate applies.

* We thank audiences at the New York Philosophy of Language Workshop, Yale University, Nagoya Gakuin University, the University of Nanjing and at SALT 26 for helpful comments and discussion. Special thanks to six anonymous SALT 26 reviewers for detailed and thought provoking written comments.

C2016 Kennedy and Willer 
(1) a. Kim finds Lee fascinating, because he is an expert on oysters.

b. \# Kim finds Lee vegetarian, because the only animals he eats are oysters.

In this sense, find contrasts with the otherwise similar verb consider, which can be used with predicates like vegetarian (as well as fascinating):

(2) a. Kim considers Lee fascinating, because he is an expert on oysters.

b. Kim considers Lee vegetarian, because the only animals he eats are oysters.

At the same time, consider is like find in rejecting fully objective predicates (Fleisher 2013):

(3) a. \# Kim finds the sum of two and two equal to four.

b. \# Kim considers the sum of two and two equal to four.

Intuitively, the sentences in (1-3) all imply that it is somehow 'up to Kim' whether the predicate in the complement can be truthfully applied to its argument (albeit in slightly different ways, as shown by the contrast between (1b) vs. (2b)), which accords with our understanding of the meaning and use of fascinating and vegetarian, but not equal to four. It is in this sense that both find and consider express subjective attitudes, and it is in this sense that they differ from a 'vanilla' doxastic attitude verb like believe, which accepts any kind of predicate in its complement:

(4) a. Kim believes that Lee is fascinating, because he is an expert on oysters.

b. Kim believes that Lee is vegetarian, because the only animals he eats are oysters.

c. Kim believes that the sum of two and two is equal to four.

The goal of this paper is to develop a semantics for subjective attitude verbs (SAVs) that captures this intuitive characterization of their difference from plain doxastic attitude verbs, and also captures the finer-grained differences between findtype SAVs and consider-type SAVs that make the former more restrictive than the latter. Pace the seminal analysis in Sæbø 2009, we do not model the restrictiveness of SAVs as a constraint on the semantic type of its complement, for reasons to be stated momentarily. Instead, we propose that their felicitous use in discourse presupposes the existence of distinct contextually provided discourse alternatives that arise from users' awareness of the contingency of how contextual underdetermination of meaning is resolved. We label these discourse alternatives COUNTERSTANCES.

Our discussion is structured as follows. We first motivate and articulate our proposal in informal terms $(\$ 2)$ and then present the formal details of our proposal $(\S 3)$. 
The resulting analysis explains the variable acceptability of predicates under find and consider. In $\S 4$, we discuss its implications for another important observation about SAVs: that find-attributions require that the attributee stand in a distinguished direct acquaintance relation to the item under consideration. $\$ 5$ concludes the discussion and points to future research.

\section{Counterstance}

In this section, we lay out the conceptual foundations of our proposal. We state our reasons for looking for an alternative to Sæbø's (2009) analysis and outline a crucial source of inspiration for our own approach: von Fintel \& Gillies's (2010) analysis of epistemic must (\$2.1). We then describe our basic account and explain its key concepts — including the one of a COUNTERSTANCE — in informal terms (§2.2).

\subsection{Motivation and inspiration}

Sæbø's (2009) analysis of find-type SAVs assigns semantic values relative to an index of evaluation (for our purposes a possible world) and lets subjective predicates have an implicit judge argument that may be set by the subject of a find-construction:

$$
\begin{aligned}
& \text { a. } \llbracket \text { find } \rrbracket^{c, w}=\lambda \phi_{\langle e, t\rangle} \lambda x . \phi(x) \\
& \text { b. } \llbracket \text { fascinating } \rrbracket^{c, w}=\lambda x \lambda y \cdot x \text { is fascinating to } y \text { at } w \\
& \text { c. } \llbracket \text { vegetarian } \rrbracket^{c, w}=\lambda x . x \text { is vegetarian at } w
\end{aligned}
$$

This proposal explains the contrast between (1a) and (1b) in terms of a of a difference in semantic type. Specifically, 'Lee (is) vegetarian' is of not of the right type to serve as an argument for find, while 'Lee (is) fascinating' is.

A considerable drawback of Sæbø's analysis is that it does not easily generalize so that it addresses the more fine-grained distinction between find-and consider-type SAVs. Specifically, there is little evidence that the restrictiveness of the latter is amenable to an explanation in terms of semantic type. Consider, for instance, the contrast between (6a) and (6b):

(6) a. \# Kim considers Burgundy part of France.

b. Kim considers Crimea part of Russia.

The intuitive explanation of the contrast is that the sovereignty over Crimea is disputed, hence the use of consider in (6b) seems appropriate, while Burgundy being part of France would count as an objective fact, hence the use of consider in (6a) appears odd. But this is not a difference of semantic type; it is simply a matter of what background information the discourse context provides: it is hard to see 
how 'Burgundy (is) part of France' and 'Crimea (is) part of Russia' could differ in semantic type in the first place.

Nothing said so far rules out that the restrictiveness of find-and consider-type SAV s could receive radically different explanations, one being semantic à la Sæb $\varnothing$ (2009) and the other being essentially pragmatic in spirit. Insofar as we are interested in a uniform explanation of the data, however, the previous considerations suggest that the selectiveness of SAVs is best understood as a constraint on the discourse context, and it is this strategy that we are going to pursue here.

Our proposal owes inspiration to the discussion of epistemic must by von Fintel \& Gillies (2010). The classical quantificational analysis of epistemic must as a universal quantifier over possibilities compatible with what is known predicts that ' $m u s t \phi$ ' entails $\phi$ : since whatever is known is true, the actual world must be among the possibilities over which must quantifies. But this, as Karttunen (1972) observes, clashes with the basic intuition that 'It must be raining' says something weaker than plain 'It is raining.' Pace Kratzer (1991) and Veltman (1985), whose semantic analyses block the inference of $\phi$ from 'must $\phi$,' von Fintel and Gillies aim to show that Karttunen's observation is in fact compatible with the predictions of the classical quantificational analysis of epistemic must. Their proposal is that in addition to its universal quantificational force, epistemic must carries with it an additional evidential component, such that an utterance of ' $m u s t ~ \phi$ ' is appropriate only if the prejacent is known on the basis of indirect evidence or deduction, rather than on the basis of direct evidence.

To illustrate, if Alice is looking out of the window seeing pouring rain, she can assert (7a) but not (7b):
a. It is raining.
b. It must be raining.

If instead she sees people coming from outside with wet raingear, both (7a) and (7b) are acceptable, even if it is clear that rain is the only possible explanation. Differences in context together with the evidential dimension of epistemic must explain this contrast: in the second scenario, but not in the first, does Alice infer that it is raining.

We claim that something along these lines is also right for find and consider. In terms of their core at-issue content, these verbs are just like believe in expressing a doxastic attitude toward the prejacent: find-and consider-statements entail the corresponding believe-statements. What makes SAVs special is that they presuppose the contingency of their prejacent with respect to a class of contextually generated discourse alternatives. Let us explain. 


\subsection{Our proposal}

We start with the standard treatment of possible worlds as first-order models that assign to $n$-ary predicates of our target language sets of $n$-tuples as their extension, and note that at this level of analysis there is no difference between 'objective' and 'subjective' predicates insofar as both have extensions relative to possible worlds. We also follow standard protocol in assuming that at least one aspect of the discourse context is to be modeled by the context set, that is, the set of possible worlds compatible with what is common ground between the discourse participants (see Stalnaker 1978). In actual discourse interactions, participants strengthen the common ground both by exchanging factual information and through other discourse moves such as explicit or implicit agreement about application criteria (e.g., by deciding to exclude eating mollusks from the factors that would disqualify an individual from counting as vegetarian), by coordinating on evaluative judgments or tastes, and by other means. Importantly, our setup models all of these effects as restrictions on the context set, since they all amount to ruling out certain possible worlds qua first-order models from consideration.

In the previous paragraph we alluded to the distinction between 'objective' and 'subjective' predicates but also said that it does not correspond to a distinction at the level of possible worlds or of conversational dynamics. Instead, we propose that it corresponds to a pragmatic distinction between those aspects of the common ground that discourse participants take be grounded in objective facts of the world and those they take to be (to a certain extent) arbitrary matters of linguistic practice. To model this distinction, we embellish our context model with a function $\kappa$ that tracks the contingency of the stipulations involved in achieving an information state. $\kappa$ takes an information carrier $s$ and derives a set $\kappa(s)$ of $s$ 's COUNTERSTANCES: each such counterstance agrees with $s$ on its factual information but disagrees on contextually salient decisions about linguistic practice. So for instance, a state $s$ and its counterstances may disagree on what it takes to be vegetarian (whether or not eating mollusks disqualifies one from being vegetarian, for instance) and thus on whether Lee is in the extension of vegetarian, but will not disagree on matters of fact such as whether Lee eats oysters.

The setup outlined so far allows us to distinguish between predicates whose extension varies across an information state and its counterstances and those whose extension does not, and we propose that it is this difference that captures the basic distinction between 'objective' and 'subjective' predicates: the extensions of objective predicates remain constant under the counterstance generating operation; the extensions of subjective predicates do not. Relatedly, we can say that a proposition is subjective just in case we can find some information state $s$ that accepts it - the proposition is true at all possible worlds in $s$ - but that fails to be accepted by one 
of $s$ 's counterstances. We label this COUNTERSTANCE CONTINGENCY.

In light of the previous remarks, our basic proposal - subject to refinement below as we spell out the formal details - is that SAVs presuppose the counterstance contingency of their complements. SAVs reject complements based on predicates like equal to four because such predicates are objective in the sense defined above, and so the truth value of a proposition based on such a predicate in an information state $s$ remains the same in s's counterstances. In contrast, the truth value of propositions based on predicates like vegetarian and fascinating in an information state $s$ do not carry over to all of $s$ 's counterstances, because the criteria that determine what it takes to be vegetarian or fascinating (as well as those that determine how fascinating something is) are subject to variation and negotiation. Different choices result in different extensions for the predicates across counterstances, and corresponding variation in truth values for the propositions they are used to express. The proposal also explains why it is odd to consider Burgundy part of France but alright to consider Crimea part of Russia: in light of current events, certain salient metalinguistic decisions will matter for whether 〈Crimea, Russia is in the extension of part of, but they do not matter for whether $\langle$ Burgundy, France $\rangle$ is.

In order to capture the further distinction between find-and consider-type SAVs, we build on our earlier observation that discourse participants strengthen the common ground both through the exchange of factual information and also by coordinating on an array of parameters that are relevant for setting predicate extensions and are subject to inter-speaker variation, including those that matter for the extension of predicates such as vegetarian and fascinating. Our proposal is compatible with different formalizations of these features - more on this at a later stage - but relies on there being a distinction between those discourse parameters that support COORDINATION BY STIPULATION and those that do not.

As a way of motivating this distinction, we first note that statements like (8a-b) are perfectly natural while $(9 \mathrm{a}-\mathrm{b})$ sound odd:

(8) For the purposes of this discussion ...

a. ... let's count Lee as vegetarian, since the only animals he eats are oysters.

b. $\quad .$. let's count these oysters as expensive, because they cost $\$ 36$ per dozen.

(9) For the purposes of this discussion ...

a. ?? ... let's count Lee as fascinating, since he is an expert on oysters.

b. ?? ... let's count these oysters as tasty, because of their texture and brine.

These contrasts suggest that certain contextual parameters can be fixed via stipulative discourse moves, such as what kinds of eating habits should be taken into 
account when considering whether an individual is vegetarian and what degree of cost determines whether something is expensive, while others cannot be, or at least not in a natural way, such as what kind of characteristic makes someone fascinating or what kind of texture and flavor makes something tasty. We model this distinction by introducing a second contextual function $\kappa_{c}^{*}$ that partitions $\kappa_{c}(s)$ - the set of counterstances of an information carrier $s$-into a set of equivalence classes of counterstances that agree on parameters that support coordination by stipulation. Intuitively, $\kappa_{c}(s)$ provides the full set of (contextually salient) resolutions of uncertainty of meaning and use in $s$, while $\kappa_{c}^{*}\left(\kappa_{c}(s)\right)$ structures those resolutions into the ones that can be naturally stipulated in discourse, which vary across equivalence classes, and those that cannot be, which vary within equivalence classes.

We propose that the difference between find and consider is that the latter presupposes mere counterstance contingency, while the former introduces the stronger presupposition that the prejacent is counterstance contingent relative to every partition introduced by $\kappa_{c}^{*}$, i.e. no matter how we resolve uncertainty of meaning based on parameters that support coordination by stipulation. This presupposition is satisfied in (10a), because the application conditions for a predicate like fascinating are at least partly determined by experiences of interest/engagement/curiosity, which resist being brought into agreement by simple stipulation.
a. Kim finds Lee fascinating.
b. \# Kim finds Lee vegetarian.

In contrast, the application conditions for a predicate like vegetarian are subject to stipulation, as we have seen. The counterstance space can be partitioned based on particular choices about how to resolve them, and within each partition the truth of the prejacent in (10b) is invariant, depending on Lee's actual eating habits. The result is that the presupposition of find which requires counterstance contingency relative to every partition is not satisfied, even though the weaker presupposition of mere counterstance contingency imposed by consider is satisfied.

So far we have offered our motivation for adopting a pragmatic approach to SAVs and the distinction between subjective and objective language more generally, and outlined the key maneuvers of our proposal as well as their key inspiration. Before formalizing the proposal, we want to highlight how some of its main ideas compare to other recent trends in the linguistics literature on subjectivity. Perhaps most importantly, our treatment of a possible worlds as determining extensions for 'objective' and 'subjective' predicates alike is sympathetic to Coppock's (2016) recent proposal to replace possible worlds with outlooks, which do not only settle matters of fact but also matters of opinion. One important difference, however, is that Coppock presupposes a categorical distinction between objective and nonobjective predicates: as Coppock defines them, outlooks 'refine' a possible world in 
the sense that they agree on all the objective predicate extensions but, in addition, say something about the non-objective ones as well. Importantly, we do not think that such a categorical distinction can be drawn: what counts as a subjective predicate is context-sensitive and depends on which counterstances discourse makes available. Furthermore, Coppock's proposal does not capture the two types of subjectivity that emerges in the variable acceptability of predicates under find and consider, and thus does not explain the finer-grained distinction between find-and consider-type SAVs that is so central to our discussion here.

We also wish to note that our presuppositional analysis of the selectional properties of SAVs fits squarely within a broader set of analyses of the selectional properties of modals and attitude verbs that are based on the idea that such expressions introduce different kinds of contingency or 'non-settledness' conditions on their prejacents. Examples include, in addition to von Fintel and Gillies's (2010) analysis of epistemic must, Condoravdi's (2002) analysis of the distribution of future-oriented interpretations of possibility modals, Giannakidou and Mari's (2016) analysis of the future as an epistemic modal, and Giannakidou and Mari's (2015) analysis of the distribution of indicative vs. subjunctive mood in the complements of emotive attitude predicates. Our analysis bears a particularly close conceptual connection to Bouchard's (2012) analysis of find-type SAVs, who suggests that such expressions carry what he labels a 'subjective contingency presupposition' (see also Fleisher 2013). Indeed, Bouchard's informal characterization of this presupposition is similar to what we said about counterstance contingency: keeping all the non-subjective facts constant, it must be possible to judge the complement clause true, and it must be possible to judge it false. However, Bouchard's implementation of his proposal is importantly different from ours: using Coppock's terminology, it effectively translates into the claim that for a find-attribution to have a truth-value at some possible world $w$, the prejacent must be contingent across the set of outlooks refining $w$. Again, this proposal by itself does not explain the difference between find-and consider-type SAVs, and draws a semantic distinction between subjective and objective predicates, whereas we claim that this distinction is best understood as pragmatic in nature.

\section{Formal details}

In this section we lay out the formal details of the proposal. To get the analysis into clearer view, we start with a simplified proposal that makes adequate predictions for attitude ascriptions whose prejacents are atomic (\$3.1). In $\$ 3.2$ we refine the analysis so that it captures some central observations about more complex attitude ascriptions. 
Counterstance contingency

\subsection{Core analysis}

We begin by defining the basic notions of a possible world and of an information state. Given an underlying domain of individuals $D$, a possible world $w$ maps (i) each constant of our target language $\mathscr{L}$ to its world-invariant denotation $d \in D$ and (ii) each $n$-place predicate of $\mathscr{L}$ to some set of $n$-tuples of objects from $D$ (for each $n \in \mathbb{N}) . W$ is the set of all possible worlds, and $\mathscr{P}(W)$ is the set of sets of possible worlds. An information state $s \subseteq W$ is just a set of possible worlds and we let $S=\mathscr{P}(W)$ denote the set of all information states.

We assume that our semantic models provide some doxastic accessibility relation. Fix some subset $D_{a} \subseteq D$ as the set of doxastic agents. Then Dox: $\left(D_{a} \times W\right) \mapsto \mathscr{P}(W)$ assigns to each doxastic agent $a$ and possible world $w$ the set of possible worlds compatible with what $a$ believes at $w$. We define the standard truth-conditions for belief attributions on that basis (here and throughout we assume $w(\alpha)$ - the denotation of the constant $\alpha$ at $w$ - denotes a proper doxastic agent):

$$
\llbracket \alpha \text { believes } \phi \rrbracket^{c, w}=1 \text { iff } \operatorname{Dox}(w(\alpha), w) \subseteq \llbracket \phi \rrbracket^{c}
$$

As stated earlier, we assume that context $c$ fixes not only a context set $s_{c}$ representing what is common ground but also provides two functions $\kappa_{c}$ and $\kappa_{c}^{*}$. The former determines, for each state of information $s$ in its domain, a set of counterstances $\kappa_{c}(s)$ (we allow for $\kappa$ to be partial since not all information states will always matter for determining the counterstance contingency of a proposition); the latter partitions each $\kappa_{c}(s)$ into a set of equivalence classes in which all parameters that support coordination by stipulation are the same.

i. The context set $s_{c} \subseteq W$ is the set of possible worlds compatible with what is common ground between the discourse participants in $c$.

ii. $\kappa_{c}: \mathscr{P}(W) \mapsto_{p} \mathscr{P}(\mathscr{P}(W))$ maps selected $s \subseteq W$ to the set of worlds just like $s$ except for contextually salient decisions about how to resolve indeterminacy of meaning; every $s^{\prime} \in \kappa_{c}(s)$ is a COUNTERSTANCE of $s$ with respect to $c$.

iii. $\kappa_{c}^{*}: \mathscr{P}(\mathscr{P}(W)) \mapsto_{p} \mathscr{P}(\mathscr{P}(\mathscr{P}(W)))$ PARTITIONS $\kappa_{c}(s)$ into a set of counterstances so that in every partition $\pi$ every parameter that supports coordination by stipulation is held constant.

Given some context $c$ we can then define two senses in which a proposition $p$ may be counterstance contingent:

i. A proposition $p \subseteq W$ is counterstance contingent in context $c$ iff $\exists s \in S \exists s^{\prime} \in$ $\kappa_{c}(s): s \subseteq p \& s^{\prime} \nsubseteq p$. 
ii. A proposition $p \subseteq W$ is radically counterstance contingent in context $c$ iff $\exists s \in S: s \subseteq p \& \forall \pi \in \kappa_{c}^{*}\left(\kappa_{c}(s)\right) \exists s^{\prime} \in \pi: s^{\prime} \nsubseteq p$

It follows immediately from these definitions that whenever a proposition is radically counterstance contingent, it is also counterstance contingent, but not vice versa.

The basic proposal then is that consider and find are in their core at issue content just like believe: they express doxastic attitudes. In addition, the former presupposes the counterstance contingency of its prejacent, while the latter presupposes the radical counterstance contingency of its prejacent. The following articulate these claims more precisely, assuming here that $\phi$ is atomic:

a. $\llbracket \alpha$ considers $\phi \rrbracket^{c, w}$ is defined only if $\llbracket \phi \rrbracket^{c}$ is counterstance contingent in context $c$.

b. If defined, then $\llbracket \alpha$ considers $\phi \rrbracket^{c, w}=1$ iff $\operatorname{Dox}(w(\alpha), w) \subseteq \llbracket \phi \rrbracket^{c}$

a. $\quad \llbracket \alpha$ finds $\phi \rrbracket^{c, w}$ is defined only if $\llbracket \phi \rrbracket^{c}$ is radically counterstance contingent in context $c$.

b. If defined, then $\llbracket \alpha$ finds $\phi \rrbracket^{c, w}=1$ iff $\operatorname{Dox}(w(\alpha), w) \subseteq \llbracket \phi \rrbracket^{c}$

Thus find-and consider-statements entail the corresponding believe-statements. Furthermore, since every radically counterstance contingent proposition is counterstance contingent (but not vice versa) we predict that whenever a find-statement is felicitous, so is its corresponding consider-statement (but not vice versa).

It is easy to verify that everything said here captures what we proposed earlier. Specifically, in ordinary contexts $c$, Lee is vegetarian $\rrbracket^{c}$ is counterstance contingent but not radically counterstance contingent, while $\llbracket$ Lee is fascinating $\rrbracket^{c}$ is radically counterstance contingent. This is why simple predications of vegetarian are acceptable under consider but not under find, and why simple predications involving fascinating are acceptable under the scope of both types of SAVs. Relatedly, «Burgundy is part of France $\rrbracket^{c}$ fails to be counterstance contingent, while $\llbracket$ Crimea is part of Russia $\rrbracket^{c}$ is counterstance contingent (but not radically so), which explains why the latter, but not the former, is acceptable under the scope of consider (but not find).

In the next subsection, we highlight some limitations of the basic proposal and outline a refined analysis that overcomes these difficulties.

\subsection{Refinement}

While the basic proposal is adequate as long as the prejacent of the relevant attitude ascription is atomic, it runs into difficulties once the prejacent is complex. Here we consider two key observations that are inspired by Sæbø (2009). Note that there is a striking contrast between (14a) and (14b), and also between (14c) and (14d). 
Counterstance contingency

(14) a. Kim finds Lee handsome and pleasant to be with.

b. \# Kim finds Lee handsome and under 45.

c. Kim finds everyone who is not vegetarian unpleasant.

d. \# Kim finds everyone who is pleasant vegetarian.

Our basic proposal does not predict the observed contrasts: a conjunctive proposition is (radically) counterstance contingent just in case at least one of its conjuncts is, and so (14a) and (14b) should both be fine. Relatedly, under the reasonable assumption that 'everyone who is not vegetarian is unpleasant' and 'everyone who pleasant is vegetarian' are truth-conditionally equivalent, our basic proposal predicts that (14c) and $(14 \mathrm{~d})$ are equally acceptable, contrary to the facts.

Sæbø (2009) once again appeals to a type difference between subjective and objective predicates to explain the contrasts. Due to their type difference, handsome and under 45 cannot be coordinated unless a covert pronoun fills the judge position of the former. But then the coordinated structure handsome and under 45 fails to be judge-dependent and thus does not embed felicitously under find, predicting that (14b) is marked (since no judge position needs to be filled to coordinate handsome and pleasant to be with, we expect (14a) to be fine). For (14c), Sæbø assumes that the subject DP quantifier raises, leaving unpleasant with an open judge argument when it composes with find, as required. In (14d), quantifier raising will leave nothing judge dependent to combine with find, and even if there is no raising, the judge argument of pleasant would need to be filled to allow for composition with the determiner phrase.

For current purposes, we set aside specific concerns about Sæbø's analysis and focus on our key worry, which again pertains to the challenge of arriving at a suitably general explanation of what distinguishes SAVs from plain doxastic attitude verbs. Note that consider-type SAVs exhibit a pattern similar to find-type SAVS.

(15) a. Kim considers Lee vegetarian and intelligent.

b. \# Kim considers Lee vegetarian and in the cast of Hamilton.

c. Kim considers someone who is in the cast of Hamilton vegetarian.

d. \# Kim considers someone who is vegetarian in the cast of Hamilton.

A type theoretic explanation of these contrasts à la Sæbø would need to assign to vegetarian a type so that this expression - unlike in the cast of Hamilton - embeds felicitously under consider but - unlike fascinating — fails to embed felicitously under find. For reasons stated earlier, it is hard to see what could motivate such a maneuver.

But the fact remains that if we simply look at the (radical) counterstance contingency of the prejacent, we will not be able to explain the observed contrasts in 
acceptability: if a proposition $p$ is counterstance contingent, then the conjunction of $p$ and $q$ should be as well. Here we aim for a uniform explanation that starts with the intuition that the complement of an attitude ascription not only expresses a proposition but also highlights a set of issues. For instance, to say that Kim finds Lee handsome and pleasant to be with is just to say that Kim's attitude speaks to the issue of whether Lee is handsome, and also to the issue of whether Lee is pleasant to be with. If we now say that SAVs presuppose that each of the issues raised by the prejacent allows for a (radically) counterstance contingent resolution, it follows that (14a) and (15a) are fine while (14b) and (15b) are marked.

The outlined strategy for expanding the basic analysis to complex attitude ascriptions - by looking at the issues raised by the complement of the attitude ascription under consideration - also leads to a plausible explanation of the previous observations about quantifiers in the scope of SAVs. The proposal is that quantified constructions raise the material in their scope, but not the material in their restrictor, as an issue. Specifically, to say that Kim finds everyone who is not vegetarian unpleasant is to say that Kim's attitude speaks to the issue of who is unpleasant - an issue that allows for a radically counterstance contingent resolution. To say that Kim finds everyone who is pleasant vegetarian is to say that Kim's attitude speaks to the issue of who is vegetarian - an issue that fails to allow for a radically counterstance contingent resolution. Clearly, this explanatory strategy not only addresses the contrast between the find-constructions in (14c) and (14d) but also easily generalizes so that it explains the observed contrast between (15c) and (15d) involving consider.

It is straightforward to spell out the formal details of this proposal. Taking some inspiration from Groenendijk \& Stokhof (1984), we associate with each atomic question its set of true and complete answers, and treat this set as the issue raised by the corresponding atomic sentence, each element of that set counting as a possible resolution of the issue. We then derive the issues raised by complex sentences compositionally on the basis of the issues raised by their components.

Since our analysis now includes quantifiers, let us assume explicitly that our language provides a set of variables $x, y, z, \ldots$ and that context fixes a variable assignment $g_{c}$. We say that $\operatorname{Alt}(c)$ is the set of contexts just like $c$ except for their variable assignments. If $\alpha$ is a singular expression, then $i_{c}(\alpha)$ - the denotation of $\alpha$ in $c$ - is $w(\alpha)$ in case $\alpha$ is some constant of $g_{c}(\alpha)$ in case $\alpha$ is a variable. As usual we say that $\llbracket F \alpha_{1} \ldots \alpha_{n} \rrbracket^{c, w}=1$ iff $\left\langle i_{c}\left(\alpha_{1}\right), \ldots, i_{c}\left(\alpha_{n}\right)\right\rangle \in w(F)$. We can then define an operation '?' on atomic sentences as follows:

$$
\llbracket F \alpha_{1} \ldots \alpha_{n} ? \rrbracket^{c, w}=\left\{w^{\prime}: \llbracket F \alpha_{1} \ldots \alpha_{n} \rrbracket^{c^{\prime}, w}=\llbracket F \alpha_{1} \ldots \alpha_{n} \rrbracket^{c^{\prime}, w^{\prime}} \text { for all } c^{\prime} \in \operatorname{Alt}(c)\right\}
$$

The semantic value of ' $F \alpha_{1} \ldots \alpha_{n}$ ?' at some possible world $w$ is thus the set of possible worlds in which the same $n$-tuples of individuals satisfy $\phi$ as in $w$.

It is then straightforward to associate with each sentence a set of issues that it 
raises given some context $c$. Here we let $Q$ represent a determiner that may bind variables at the level of logical form.

$$
\begin{array}{ll}
\text { a. } & \mathscr{I}_{c}\left(F \alpha_{1} \ldots \alpha_{n}\right)=\left\{\llbracket F \alpha_{1} \ldots \alpha_{n} ? \rrbracket^{c}\right\} \\
\text { b. } & \mathscr{I}_{c}(\neg \phi)=\mathscr{I}_{c}(\phi) \\
\text { c. } & \mathscr{I}_{c}(\phi \vee \psi)=\mathscr{I}_{c}(\phi \wedge \psi)=\mathscr{I}_{c}(\phi) \cup \mathscr{I}_{c}(\psi) \\
\text { d. } & \mathscr{I}_{c}(Q(\phi, \psi))=\mathscr{I}_{c}(\psi)
\end{array}
$$

So for instance, an atomic sentence just raises the question associated with it as an issue. 'Lee is vegetarian and intelligent' raises the issue of whether Lee is vegetarian and the issue of whether Lee is intelligent. A quantified sentence such as 'Everyone who is vegetarian is intelligent' raises the issue of who is intelligent.

It then makes sense to extend the definition of (radical) counterstance contingency to sets of issues, as follows. To say that an issue is (radically) counterstance contingent is to say that one of its resolutions is (radically) counterstance contingent. A set of issues is (radically) counterstance contingent just in case each of its members is (radically) counterstance contingent. We may then refine the analysis of find and consider as follows:

(18) a. $\quad \llbracket \alpha$ considers $\phi \rrbracket^{c, w}$ is defined only if $\mathscr{I}_{c}(\phi)$ is counterstance contingent in the context $c$.

b. If defined, then $\llbracket \alpha$ considers $\phi \rrbracket^{c, w}=1$ iff $\operatorname{Dox}(w(\alpha), w) \subseteq \llbracket \phi \rrbracket^{c}$

a. $\quad \llbracket \alpha$ finds $\phi \rrbracket^{c, w}$ is defined only if $\mathscr{I}_{c}(\phi)$ is radically counterstance contingent in the context $c$.

b. If defined, then $\llbracket a$ finds $\phi \rrbracket^{c, w}=1$ iff $\operatorname{Dox}(w(\alpha), w) \subseteq \llbracket \phi \rrbracket^{c}$

It is easy to verify that the formal analysis captures what we proposed earlier. Consider again the earlier observed contrasts involving find, repeated below:

(14) a. Kim finds Lee handsome and pleasant to be with.

b. \# Kim finds Lee handsome and under 45.

c. Kim finds everyone who is not vegetarian unpleasant.

d. \# Kim finds everyone who is pleasant vegetarian.

In ordinary contexts $c$, «Lee is under $45 \rrbracket^{c}$ fails to be radically counterstance contingent, and so the set of issues raised by 'Lee is handsome and under 45' fails to be radically counterstance contingent. So we expect (14b) to be marked. (14a), in contrast, is fine since both $\llbracket$ Lee is handsome $\rrbracket^{c}$ and $\llbracket$ Lee is pleasant to be with $\rrbracket^{c}$ exhibit radical counterstance contingency. For parallel reasons, the issue of who 
is vegetarian fails to be radically counterstance contingent, explaining why (14d) is unacceptable, while the radical counterstance contingency of the issue of who is unpleasant licenses (14c).

We conclude that our basic analysis can be refined so that it makes correct predictions about the acceptability of coordinated and quantified constructions under the scope of find-type SAVs. Importantly, the refinement relies on a single and intuitive idea - that ordinary sentences raise issues whose resolutions may or may not be radically counterstance contingent - and it immediately generalizes so that it explains corresponding observations about consider-type SAVs. All of this should give us some confidence that the proposal deserves to be explored further.

\section{Direct experience}

Consider a context in which Kim presents her two cats with a new variety of cat food. Hoshi, who eats anything, quickly devours the food with apparent enjoyment. Nikko, who is very picky and only eats things that she really likes, takes one sniff and walks away. Kim observes Nikko's behavior and says 'This new food is not tasty.' Both (20a-b) are felicitous as descriptions of this situation, but (20c) is not.

(20) a. Kim doesn't believe the new food is tasty, because Nikko didn't eat it.

b. Kim doesn't consider the new food tasty, because Nikko didn't eat it.

c. \# Kim doesn't find the new food tasty, because Nikko didn't eat it.

Intuitively, (20c) is strange because the use of find communicates that Kim's judgment about the taste of the new catfood is based on direct experience — she has tasted it herself — in which case using Nikko's behavior as a justification for the claim about Kim's attitude is incongruent. The fact that $(20 \mathrm{a}-\mathrm{b})$ are not odd demonstrate that neither believe nor consider introduce the same direct experience inference.

Stephenson (2007) presents an analysis of find that is similar to the one we have advocated here in treating find as a doxastic attitude verb and distinguishing it from believe in terms of additional content, which she characterizes as a further entailment that the attitude holder has direct experience of the prejacent. The fact that the direct experience inference is retained under negation in (20c) (as well as in questions and other entailment-blocking contexts) indicates that it is actually a presupposition, suggesting a variant of Stephenson's analysis which is formally just like the analysis we have developed in this paper, except that the radical counterstance contingency presupposition is replaced with a direct experience presupposition:

(21) a. $\quad \llbracket \alpha$ finds $\phi \rrbracket^{c, w}$ is defined only if $\alpha$ has direct experience of $\llbracket \phi \rrbracket^{c}$ in $w$.

b. If defined, then $\llbracket \alpha$ finds $\phi \rrbracket^{c, w}=1$ iff $\operatorname{Dox}(w(\alpha), w) \subseteq \llbracket \phi \rrbracket^{c}$ 
This raises two questions. First, is (21) a viable alternative analysis of find, or are there independent reasons to prefer an analysis based on counterstance contingency? And second, if the counterstance contingency analysis is to be preferred, what does it have to say about the direct experience presupposition? Is there a principled reason why this presupposition is present with find but not with consider (or believe), or does this difference need to be stipulated?

Regarding the first question, Sæbø (2009) has already shown that an analysis of find as a doxastic attitude verb with an additional direct experience presupposition is not sufficient to explain the constraints it imposes on its prejacent. For example, assuming that direct experience is involved in an individual's assessment of propositions pertaining to his or her own eating habits, the direct experience presupposition is satisfied in (22a-b), yet find is bad but consider is good.

a. \# Lee finds himself vegetarian (even though he eats oysters).

b. Lee considers himself vegetarian (even though he eats oysters).

One possible response to this objection is to say that the prejacents in (22a) and the similar examples that Sæbø discusses do not, after all, express propositions that one can have direct experience of, and indeed Stephenson admits that what exactly it means for an individual to have direct experience of a proposition is unclear. But this explanation runs the risk of begging the question: why is it that propositions about e.g. an individual's taste experiences are accessible to direct experience but propositions about an individual's eating habits are not? A non-question begging option would be to correlate the possibility of direct experience with an actual experiencer argument in the lexical semantics of the embedded predicate, and there are good reasons to assume that at least predicates like tasty, fun and other predicates of personal taste have such arguments. However, as shown by Bylinina (forthcoming), there are predicates that are acceptable under find, such as evaluative adjectives like charming, lazy, ugly, brave and so forth (Bierwisch 1989), which, according to various linguistic tests, such as the distribution of dative arguments in Russian and Japanese, lack experiencer arguments. We therefore conclude with Sæbø that a direct experience presupposition alone is not enough to explain the restriction of the complement of find to predicates that are (in our terms) radically counterstance contingent.

Turning to the second question, we believe that our analysis of subjective attitude verbs can, in fact, derive the direct experience requirement introduced by find as a pragmatic presupposition. According to our analysis, find semantically presupposes that its prejacent is radically counterstance contingent, which is to say that it is contingent across all partitions of the relevant counterstance space in which the parameters of meaning that support coordination by stipulation are fixed. In order to satisfy this presupposition, it must be the case that the meaning parameters that 
resist coordination by stipulation and on which the truth of the prejacent depend are indeterminate in the context, since fixing these parameters to a particular taste, experience, stance, perspective, or 'judge' (to use the term most familiar from the literature) eliminates variability in the truth of the prejacent within each partition of the counterstance space. This is why, on our account, (23a) is bad but (23b) is acceptable.
a. \# Kim finds Lee fascinating only to her(self)/Pat.
b. Kim considers Lee fascinating only to her(self)/Pat.

In a typical context, fascinating to $X$ is counterstance contingent, because there is uncertainty about the precision to which the predicate applies or about the degree of fascination that is sufficient to qualify as fascinating. But it is not radically counterstance contingent, because there is no uncertainty about the experiential criteria relevant for the application of the predicate: they are the ones determined by the denotation of $X$.

To say that proposition is presupposed to be radically counterstance contingent then, is to say that neither the facts of the world nor the conventions of linguistic practice that support coordination by stipulation provide a basis for asserting or denying the prejacent, and further that the experiential/perspectival factors relevant for evaluating its truth are indeterminate. Given this degree of uncertainty, what could provide the evidential basis for an assertion or denial of the at-issue component of the meaning of a find statement, namely that the attitude holder believes the radically counterstance contingent proposition expressed by the prejacent? The evidential basis for such an assertion or denial, we claim, is that the attitude holder has experience of the sort that supports their belief, which the case of e.g. tasty is experience of the taste of the relevant stuff, in the case of fun is participation in the relevant event, and so forth.

The reason that the direct experience requirement disappears with consider and believe is that these verbs do not presuppose radical counterstance contingency, and so crucially allow for 'exocentric' interpretations of subjective predicates in their prejacents. And indeed, when we say that Kim doesn't believe/consider the food (is) tasty in $(20 \mathrm{a}-\mathrm{b})$, it is precisely Nikko the cat whose tastes determine the kind of taste judgment expressed by the adjective. The evidential basis for belief in a proposition that is not radically counterstance contingent may include experience of the relevant sort, but are not limited to such experience: observation of the consequences of individual's experience may be sufficient.

If our account of the direct experience presupposition of find as an evidential condition emerging from the presupposition of (radical) counterstance contingency and the assertion of belief is on the right track, then we might expect to find an evidential component to consider as well, though not necessarily one involving 
direct experience. The following examples suggest that this is the case. in a context in which my only evidential base for belief in a proposition is (reliable) testimony, belief claims are fine, but consider claims are inappropriate.

(24) I do not know what's in this stew, but

a. \# ... I consider it vegetarian.

b. ... I believe that it is vegetarian.

(25) I do not know Lee's height, but

a. \# ... I consider him tall.

b. ... I believe that he is tall.

This contrast finds a natural explanation in our counterstance-based framework, without the need for additional lexical stipulations. (24a) and (25a) presuppose that the truth of the prejacent is sensitive to certain metalinguistic decisions: in the cases at hand, these decisions are naturally understood to concern whether certain kinds of food are compatible with a vegetarian diet and whether having a certain height is sufficient to count as tall. But of course this presupposition is only justified if one has reason to think that the facts on the ground actually leave room for conflicting stances, that is, only in case one has reason to think that the stew is 'borderline' vegetarian and that John is 'borderline' tall. And it is hard to see how one could have such a reason unless one knew something about the stew's ingredients or about John's height. So simply in virtue of the counterstance contingency presupposition that comes with consider we expect that — in ordinary contexts anyway - (24a) and (25a) will carry a distinct evidential flavor.

\section{Conclusion and outlook}

We have proposed an analysis of subjective attitude verbs as expressing counterstance contingent beliefs: beliefs that the attributor takes to be contingent upon distinct decisions about how to resolve aspects of meaning that are underdetermined by the facts of the discourse situation. Our analysis is compatible with different formalizations of the discourse parameters whose variability underwrites the relevant semantic underdetermination (e.g., relativist vs. contextualist vs. epistemicist), and indeed does not presume that they are homogenous. Indeed, if our analysis is correct, it suggests that subjectivity in language, as a general phenomenon, is not to be explained strictly in terms of any particular semantic parameter, implicit argument, or lexical underspecification, but rather emerges from language users' awareness of counterstance: that the choices they make about how to resolve these features in the course of constructing a common ground could have been different. Additionally, 
it provides a formal characterization of the 'two types' of subjectivity that emerge in the variable acceptability of predicates under find and consider, and a basis for deriving the direct experience presupposition of find as an evidential condition on belief in a radically counterstance contingent proposition. Looking ahead, we believe that essentially the same line of reasoning underlies the 'Acquaintance Principle' of Ninan (2014) and explains why unembedded assertions and denials of propositions involving subjective predicates also have a direct experience requirement (see also Pearson 2013; Bylinina forthcoming), but we leave that discussion to future work.

In our discussion, we omitted an issue that has been very prominent in the literature on subjective language: the intuition that if Kim says that Lee is fascinating and Alice responds that Lee is not fascinating, they disagree and, moreover, neither of them need be 'at fault' (see, for instance, Kölbel 2004; Lasersohn 2005; Glanzberg 2007; Stephenson 2007, 2008; Stojanovic 2007; Moltmann 2010; Sundell 2011; Barker 2013; Pearson 2013). We have set the issue of faultless disagreement aside partly because it is a non-trivial question how exactly this kind of disagreement is to be characterized in theoretical terms (see, for instance, MacFarlane 2014 for discussion), and partly because it is unclear whether the possibility of faultless disagreement has any distinct semantic implications once we allow for a sufficiently rich conception of the dynamics of conversation (see, for instance, Barker's (2013) model for negotiating contextual parameters for such a conception). Here we just point out that our concept of counterstance contingency also promises to be relevant for the issue of faultless disagreement: treating an issue as counterstance contingent is just to say that the objective facts (whatever those are, according to the conversational context) do not select for a unique resolution of that issue, and intuitively it is exactly the absence of a single correct view on an issue that underwrites intuitions of faultless disputes. One important implication of this approach is that since counterstance contingency is a matter of what alternatives a discourse context provides, we predict faultless disagreement to be an essentially context sensitive phenomenon - just like the acceptability of certain predicates in the scope of SAVs. Vardomskaya (In preparation) observes that this prediction is borne out: whether a disagreement counts as faultless is crucially dependent on context and not tied to lexical items.

A comprehensive articulation of our perspective on the nature of faultless disagreement not only requires a precise account of what this phenomenon is supposed to be. Above all, what is needed is a comprehensive story about the role that counterstances - and discourse alternatives more generally - play for the norms that govern how we assert, reject, and evaluate utterances in everyday discourse. We must leave such a story for another day, but conclude that it is a story worth telling: if the proposal developed here is on the right track, the notion of a counterstance is bound to play an important role in our best theory of linguistic meaning. 
Counterstance contingency

\section{References}

Barker, Chris. 2013. Negotiating taste. Inquiry 56(2-3). 240-257. doi:10.1080/0020174X.2013.784482.

Bierwisch, Manfred. 1989. The semantics of gradation. In Manfred Bierwisch \& Ewald Lang (eds.), Dimensional Adjectives, 71-261. Berlin: Springer.

Borkin, Ann Margaret Hopkins. 1974. Raising to object position: A study in the syntax and semantics of clause merging. Ann Arbor: University of Michigan $\mathrm{PhD}$ dissertation.

Bouchard, David-Ėtienne. 2012. Long-distance degree quantification and the grammar of subjectivity. Montreal: McGill University PhD dissertation.

Bylinina, Lisa. forthcoming. Judge-dependence in degree constructions. Journal of Semantics doi:10.1093/jos/ffw011.

Condoravdi, Cleo. 2002. Temporal interpretation of modals: Modals for the present and for the past. In David I. Beaver, Stefan Kaufmann, Brady Clark \& Luis Casillas (eds.), The Construction of Meaning, 59-88. Stanford, CA: CSLI Publishers.

Coppock, Elizabeth. 2016. Outlook based semantics. Manuscript, University of Gothenburg.

von Fintel, Kai \& Anthony S. Gillies. 2010. Must ...stay ...strong. Natural Language Semantics 18(4). 351-383. doi:10.1007/s11050-010-9058-2.

Fleisher, Nicholas. 2013. The dynamics of subjectivity. In Todd Snider (ed.), Semantics and Linguistic Theory (SALT) 23, 276-294. Ithaca, NY: CLC.

Giannakidou, Anastasia \& Alda Mari. 2015. Mixed (non)veridicality and mood choice in complement clauses: Starting with emotive verbs. Manuscript, University of Chicago and Institut Jean Nicod.

Giannakidou, Anastasia \& Alda Mari. 2016. A unified analysis of the future as epistemic modality: The view from Greek and Italian. Manuscript, University of Chicago and Institut Jean Nicod.

Glanzberg, Michael. 2007. Context, content, and relativism. Philosophical Studies 136(1). 1-29. doi:10.1007/s11098-007-9145-5.

Groenendijk, Jeroen \& Martin Stokhof. 1984. Studies on the semantics of questions and the pragmatics of answers. Amsterdam: University of Amsterdam $\mathrm{PhD}$ dissertation.

Hirvonen, Sanna. 2014. Predicates of personal taste and perspective dependence. London: UCL PhD dissertation.

Karttunen, Lauri. 1972. Possible and must. In John P. Kimball (ed.), Syntax and Semantics 1, 1-20. New York: Academic Press.

Kennedy, Christopher. 2013. Two sources of subjectivity: Qualitative assessment and dimensional uncertainty. Inquiry 56(2-3). 258-277. 
doi:10.1080/0020174X.2013.784483.

Kölbel, Max. 2004. Faultless disagreement. Proceedings of the Aristotelian Society 104(1). 53-73. doi:10.1111/j.0066-7373.2004.00081.x.

Kratzer, Angelika. 1991. Modality. In Armin von Stechow \& Dieter Wunderlich (eds.), Semantics: An International Handbook of Contemporary Research, 639650. Berlin: Walter de Gruyter \& Co.

Lasersohn, Peter. 2005. Context dependence, disagreement, and predicates of personal taste. Linguistics and Philosophy 28(6). 643-686. doi:10.1007/s10988005-0596-x.

MacFarlane, John. 2014. Assessment Sensitivity: Relative Truth and Its Applications. New York: Oxford University Press. doi:10.1093/acprof:oso/9780199682751.001.0001.

Mitchell, Jonathan Edward. 1986. The formal semantics of point of view. Amherst, MA: University of Massachusetts at Amherst PhD dissertation.

Moltmann, Friederike. 2010. Relative truth and the first person. Philosophical Studies 150(2). 187-220. doi:10.1007/s11098-009-9383-9.

Ninan, Dilip. 2014. Taste predicates and the acquaintance inference. In Todd Snider, Sarah D'Antonio \& Mia Weigand (eds.), Semantics and Linguistic Theory (SALT) 24, 290-304. Ihaca, NY: CLC.

Pearson, Hazel. 2013. A judge-free semantics for predicates of personal taste. Journal of Semantics 30(1). 103-154. doi:10.1093/jos/ffs001.

Sæbø, Kjell Johan. 2009. Judgment ascriptions. Linguistics and Philosophy 32(4). 327-352. doi:10.1007/s10988-009-9063-4.

Stalnaker, Robert C. 1978. Assertion. In Peter Cole (ed.), Syntax and Semantics 9, 315-332. New York: New York Academic Press.

Stephenson, Tamina. 2007. Towards a theory of subjective meaning. Cambridge, MA: MIT PhD dissertation.

Stephenson, Tamina. 2008. Judge dependence, epistemic modals, and predicates of personal taste. Linguistics and Philosophy 30(4). 487-525. doi:10.1007/s10988008-9023-4.

Stojanovic, Isidora. 2007. Talking about taste: Disagreement, implicit arguments, and relative truth. Linguistics and Philosophy 30(6). 691-706. doi:10.1007/s10988-008-9030-5.

Sundell, Timothy. 2011. Disagreements about taste. Philosophical Studes 155(2). 276-288. doi:10.1007/s11098-010-9572-6.

Vardomskaya, Tamara. In preparation. Subjectivity and shared evidence. Chicago: University of Chicago PhD dissertation.

Veltman, Frank. 1985. Logics for conditionals: University of Amsterdam PhD dissertation. 
Counterstance contingency

Christopher Kennedy

Department of Linguistics

University of Chicago

1010 E 59th Street

Chicago, IL 60637

ck@uchicago.edu
Malte Willer

Department of Philosophy

University of Chicago

1115 E 58th Street

Chicago, IL 60637

willer@uchicago.edu 